\title{
At the Intersection of Homophobia and Racism: Sociocultural Context and the Sexual Health of South Asian Canadian Gay and Bisexual Men
}

Trevor A Hart ( $\nabla$ trevor.hart@ryerson.ca )

OHTN Research Chair in Gay Men's Health, Department of Psychology, Ryerson University, 350 Victoria

Street, Toronto, https://orcid.org/0000-0001-5107-7452

\section{Ramraajh Sharvendiran}

Alliance for South Asian Aids Prevention

\section{Vijaya Chikermane}

Alliance for South Asian Aids Prevention

\section{Ammaar Kidwai}

Ryerson University

\section{Daniel Grace}

University of Toronto Dalla Lana School of Public Health

\section{Research}

Keywords: South Asian, men who have sex with men, gay men, sexual minority, sexual health, intersectionality, minority stress, HIV, health services, racism, homophobia, cultural norms, stress

Posted Date: September 4th, 2020

DOl: https://doi.org/10.21203/rs.3.rs-69355/v1

License: (9) (1) This work is licensed under a Creative Commons Attribution 4.0 International License. Read Full License 


\section{Abstract}

Objectives: South Asian gay and bisexual men in Canada are at risk for experiencing both intersectional structural oppressions related to homophobia and racism as well as for the acquisition of sexually transmitted infections including HIV. Our objective was to examine intersectional stigma among South Asian gay and bisexual men and its effects on their mental and sexual health.

Design: We recruited 39 South Asian gay and bisexual men in the Greater Toronto Area and interviewed them via focus groups $(n=37)$ or one-on-one interviews $(n=2)$. The facilitators used a semi-structured interview protocol covering topics about sexual health of South Asian gay and bisexual men, including their how their home backgrounds and their experiences of being South Asian in predominantly white gay spaces affected their sexual health, and how these experiences shaped their attitudes about sexual health services. Data were analysed to understand how Intersectional stigma affected perceived choices and health among South Asian GBM.

Results: Participants reported significant concerns about the effects of intersectional oppressions as South Asian gay and bisexual men, including distress about the potential social harms arising to one's family for having a gay or bisexual son. Men also described feeling excluded by the white-dominated gay community. Intersectional stigma led to loneliness and social isolation among South Asian gay and bisexual men, and limited social power in sexual relationships.

Conclusions: Findings suggest that South Asian gay and bisexual men preferred programs that focused on reducing social isolation and loneliness, as opposed to traditional safer sex programming focusing on HIV risk education.

\section{Full Text}

This preprint is available for download as a PDF.

\section{Supplementary Files}

This is a list of supplementary files associated with this preprint. Click to download.

- Hartetal.AIMDeclarationsAugust25.pdf

- HartetalAIMTable.pdf 